\title{
Slepton mass splittings and cLFV in the SUSY seesaw in the light of recent experimental results
}

\author{
A.J.R. Figueiredo ${ }^{a, b}$ and A.M. Teixeira ${ }^{b}$ \\ ${ }^{a}$ Centro de Física Teórica de Partículas, Instituto Superior Técnico, \\ Av. Rovisco Pais 1, 1049-001 Lisboa, Portugal \\ ${ }^{b}$ Laboratoire de Physique Corpusculaire, CNRS/IN2P3 - UMR 6533, \\ Campus des Cézeaux, 24 Av. des Landais, F-6317ry Aubière Cedex, France \\ E-mail: ajrf@cftp.ist.utl.pt, Ana.Teixeira@clermont.in2p3.fr
}

ABSTRACT: Following recent experimental developments, in this study we re-evaluate if the interplay of high- and low-energy lepton flavour violating observables remains a viable probe to test the high-scale type-I supersymmetric seesaw. Our analysis shows that fully constrained supersymmetric scenarios no longer allow to explore this interplay, since recent LHC data precludes the possibility of having sizeable slepton mass differences for a slepton spectrum sufficiently light to be produced, and in association to $\mathrm{BR}(\mu \rightarrow e \gamma)$ within experimental reach. However, relaxing the strict universality of supersymmetric soft-breaking terms and fully exploring heavy neutrino dynamics, still allows to have slepton mass splittings $\mathcal{O}($ few \%), for slepton masses accessible at the LHC, with associated $\mu \rightarrow e \gamma$ rates within future sensitivity. For these scenarios, we illustrate how the correlation between high- and low-energy lepton flavour violating observables allows to probe the high-scale supersymmetric seesaw.

Keywords: Rare Decays, Neutrino Physics, Supersymmetric Standard Model

ARXIV EPRINT: 1309.7951 


\section{Contents}

1 Introduction 1

2 The SUSY seesaw model 2

2.1 Flavour violation in the slepton sector 3

2.2 Slepton induced cLFV observables 4

2.3 Flavoured slepton mass differences 5

3 Numerical results and discussion $\quad 6$

3.1 mSUGRA-inspired universality $\quad 6$

$\begin{array}{ll}3.2 \text { Beyond mSUGRA-inspired universal conditions } & 11\end{array}$

4 Conclusions $\quad 15$

\section{Introduction}

Supersymmetric (SUSY) seesaw realisations offer an appealing framework to address several of the observational and theoretical shortcomings of the Standard Model (SM). Even if realised at a very high-scale (close to the grand unification scale, $M_{\mathrm{GUT}}$ ), prior to their decoupling, the new right-handed neutrino superfields induce corrections into the SUSY soft-breaking slepton terms. Since neutrino oscillations do not conserve lepton flavour, these corrections are lepton flavour violating (LFV), and can induce SUSY contributions to slepton mediated charged LFV (cLFV) observables [1].

Compared to its non-SUSY version [2-6], and in addition to accounting for neutrino masses and mixings, the high-scale type-I SUSY seesaw opens the door to a large number of cLFV observables at/below the $\mathrm{TeV}$ scale, that can be searched for in low-energy, high intensity facilities or in high-energy colliders as the LHC or a future Linear Collider (LC). Among the former one has flavour violating radiative and three body lepton decays, as well as muon-electron conversion in nuclei [7-30]; the latter are associated to the potential reconstruction of SUSY decay chains involving slepton intermediate states, and include various observables, such as for example flavoured slepton mass differences and direct flavour violating gaugino decays [31-45].

However, in the absence of SUSY discovery (and reconstruction of its fundamental Lagrangian), the contributions to the different cLFV observables allow for a wide range of predictions, as the observables are in general dependent on powers of the average SUSY scale and of the seesaw scale. While the first might be possibly known in the near future, the second cannot be directly probed, which renders these scenarios hard to test. However, when embedded into flavour blind SUSY breaking models, the type-I seesaw is the unique source of flavour violation in the lepton sector, implying that all lepton flavour violating 
observables will be correlated. The study of the synergy between different low-energy observables and/or high-energy ones proves to be a powerful tool to probe the high-scale type-I SUSY seesaw (see, for example, [34, 36, 37, 39, 41, 44]).

Since the first related analyses, important experimental developments have occurred, in a number of fronts. Firstly, $\theta_{13}$ has been measured [46-49], its value being sizeable. Regarding high-energy experiments, LHC negative searches on SUSY particles suggest a considerably heavier SUSY spectrum [50-58], which puts increasingly stronger bounds on the parameter space of constrained SUSY models. Accommodating the measured mass of the recently discovered SM-like Higgs boson [59-62] renders the latter bounds even more severe. Finally, the MEG experiment has significantly improved the upper bounds on $\mathrm{BR}(\mu \rightarrow e \gamma)$ [63]. In view of the latter developments, it is important to re-evaluate the prospects of probing the type-I SUSY seesaw via the synergy between slepton mass differences (if measured at the LHC) and low-energy cLFV observables such as $\operatorname{BR}(\mu \rightarrow e \gamma)$. Charged sleptons may indeed be discovered in the forthcoming $\sqrt{s}=14 \mathrm{TeV}$ LHC run or then in the subsequent high luminosity phase, for which an integrated luminosity $\sim 3000 \mathrm{fb}^{-1}$ is expected $[64,65]$. If indeed discovered, promising windows over the lepton flavour puzzle can be opened, with prospects for shedding light on the mechanism of neutrino mass generation. ${ }^{1}$

The aim of the present study is thus to discuss whether sleptons with inter-generational mass differences (resulting mainly from a high-scale type-I SUSY seesaw), compatible with current cLFV results and negative SUSY searches, can be seen in future LHC runs, and how such observations would in turn affect the information one could derive on the seesaw parameters. To do so, we consider the embedding of a type-I seesaw into constrained SUSY models, in particular into the constrained minimal supersymmetric standard model (cMSSM), extended by three generations of right-handed neutrino superfields. We then relax some of the cMSSM strict universality conditions for the different sectors, still preserving flavour universality. We discuss the impact of these scenarios on high-energy cLFV observables as slepton mass differences (between sleptons of different families), while at low-energies we focus on $\mu \rightarrow e \gamma$ decays and $\mu-e$ conversion in Nuclei.

The paper is organised as follows. In section 2 we briefly describe the type-I SUSY seesaw model and its most relevant phenomenological signatures. In section 3 we present the analysis and discuss the results; our conclusions are summarised in section 4 .

\section{The SUSY seesaw model}

The type-I SUSY seesaw consists of the Minimal Supersymmetric Standard Model (MSSM), extended by three generations of right-handed neutrino (chiral) superfields $\hat{N}_{i}^{c} \sim\left(\nu^{c}, \tilde{\nu}_{R}^{*}\right)_{i}$. The leptonic part of the superpotential reads

$$
\mathcal{W}^{\text {lepton }}=\hat{N}^{c} Y^{\nu} \hat{L} \hat{H}_{2}+\hat{E}^{c} Y^{l} \hat{L} \hat{H}_{1}+\frac{1}{2} \hat{N}^{c} M_{R} \hat{N}^{c},
$$

\footnotetext{
${ }^{1} \mathrm{~A}$ recent work has revisited charged cLFV signatures, within a SU(5) GUT framework, in low-energy observables and in flavour violating neutralino decays [45]. Low-energy cLFV in the framework of an $\mathrm{SO}(10)$ embedded type-I SUSY seesaw, taking into account the constraints from $m_{h}$, was discussed in [66].
} 
where $\hat{L}$ and $\hat{E}^{c}$ denote the $\mathrm{SU}(2)$ lepton doublet and right-handed charged lepton superfields, respectively, and $\hat{H}_{1,2}$ are the two Higgs supermultiplets. Without loss of generality, we work in a basis where both the charged lepton Yukawa couplings $Y^{l}$ and the Majorana mass matrix $M_{R}$ are diagonal. For completeness, ${ }^{2}$ the slepton soft breaking potential is given by

$$
\begin{aligned}
\mathcal{V}_{\text {soft }}^{\text {slepton }}= & \tilde{\ell}_{L}^{*} m_{\tilde{L}}^{2} \tilde{\ell}_{L}+\tilde{\ell}_{R}^{*} m_{\tilde{E}}^{2} \tilde{\ell}_{R}+\tilde{\nu}_{R}^{*} m_{\tilde{\nu}_{R}}^{2} \tilde{\nu}_{R} \\
& +\left(\tilde{\ell}_{R}^{*} A^{l} \tilde{\ell}_{L} H_{1}+\tilde{\nu}_{R}^{*} A^{\nu} \tilde{\nu}_{L} H_{2}+\frac{1}{2} \tilde{\nu}_{R} B_{\nu} \tilde{\nu}_{R}+\text { H.c. }\right) .
\end{aligned}
$$

We consider a flavour-blind SUSY breaking mechanism (so that the Yukawa couplings are the only source of flavour violation), as for example the case of minimal supergravity mediated SUSY breaking, assuming that the soft breaking parameters satisfy universality conditions at some high-energy scale, which we take to be the gauge coupling unification scale, $M_{\mathrm{GUT}} \sim 10^{16} \mathrm{GeV}$ :

$$
M_{i}^{\psi}=M_{1 / 2}, \quad\left(m_{\tilde{\phi}}^{2}\right)_{i j}=\delta_{i j} m_{0}^{2}, \quad\left(A_{\phi}\right)_{i j}=A_{0}^{\phi}\left(Y^{\phi}\right)_{i j} .
$$

In the seesaw limit (i.e., $Y^{\nu} v_{2} \ll M_{R}$ ), after electroweak (EW) symmetry breaking, the light neutrino mass matrix is approximately given by $m_{\nu} \simeq-v_{2}^{2} Y^{\nu T} M_{R}^{-1} Y^{\nu}$, where $v_{2}$ is one of the vacuum expectation values of the neutral Higgs $H_{i}\left(v_{1(2)}=v \cos (\sin ) \beta\right.$, with $v=174 \mathrm{GeV}$ ). As suggested from the seesaw expression for $m_{\nu}$, a convenient means of parameterising the neutrino Yukawa couplings $Y^{\nu}$, while at the same time allowing to accommodate neutrino data, is given by the Casas-Ibarra parameterisation [14]. At the seesaw scale one can write

$$
Y^{\nu}=\frac{i}{v_{2}} \sqrt{M_{R}^{\text {diag }}} R \sqrt{m_{\nu}^{\text {diag }}} U^{\mathrm{MNS}^{\dagger}},
$$

which we will use in our numerical analysis. In the above, $U^{\mathrm{MNS}}$ is the leptonic mixing matrix and $R$ is a complex orthogonal matrix, parameterised in terms of three complex angles $\left(\theta_{i}\right)$, that encodes additional mixings involving the right-handed $(\mathrm{RH})$ neutrinos; $m_{\nu}^{\text {diag }}$ and $M_{R}^{\text {diag }}$ respectively denote the (diagonal) light and heavy neutrino mass matrices.

\subsection{Flavour violation in the slepton sector}

Due to the non-trivial flavour structure of $Y^{\nu}$, the running from $M_{\mathrm{GUT}}$ down to the seesaw scale $M_{R}$ will induce flavour mixing in the otherwise (approximately) flavour conserving slepton soft breaking terms [1]. This running is more pronounced in the "left-handed" soft breaking terms (i.e., the terms involving slepton doublets). At leading order (leading logarithm (LLog) approximation), the flavour mixing induced by the renormalisation group (RG) flow reads

$$
\begin{aligned}
\left(\Delta m_{\tilde{L}}^{2}\right)_{i j} & =-\frac{1}{8 \pi^{2}}\left(m_{\tilde{L}}^{2}+m_{\tilde{\nu}_{R}}^{2}+m_{H_{2}}^{2}+\left|A_{0}^{\nu}\right|^{2}\right)\left(Y^{\nu \dagger} L Y^{\nu}\right)_{i j} \\
\left(\Delta A^{l}\right)_{i j} & =-\frac{1}{16 \pi^{2}}\left(A_{0}^{l}+2 A_{0}^{\nu}\right) Y_{i i}^{l}\left(Y^{\nu \dagger} L Y^{\nu}\right)_{i j} ; L_{k l} \equiv \log \left(\frac{M_{\mathrm{GUT}}}{M_{R_{k}}}\right) \delta_{k l} .
\end{aligned}
$$

\footnotetext{
${ }^{2}$ Since we work in a regime $M_{R} \gg m_{\text {soft }}$ the effects of the $B_{\nu}$-term (assumed to be $B_{\nu} \sim m_{\text {soft }}^{2}$ ) become negligible in comparison to the superpotential mass term $\left(\tilde{\nu}_{R}^{*} M_{R}^{2} \tilde{\nu}_{R}\right)$ - see for e.g. [67] for a discussion -, and will not be taken into account in the analysis.
} 
As is clear from the above, the amount of flavour violation in the slepton sector is encoded in $\left(Y^{\nu \dagger} L Y^{\nu}\right)_{i j}$, originating from light neutrino mixing and from possible mixings involving the heavy neutrinos (see eq. (2.4)). Having a unique source of LFV is the key to all tests of the SUSY seesaw; this becomes particularly clear in the simple (conservative) limit in which one assumes little (or no) additional mixing involving the heavy RH states (i.e., $R \sim \mathbb{1}$ ). To a good approximation, the intrinsic amount of cLFV is related to low-energy leptonic mixings as

$$
\left(Y^{\nu \dagger} L Y^{\nu}\right)_{i j} \simeq U_{i k}^{\mathrm{MNS}} U_{j k}^{\mathrm{MNS} *}\left(m_{k} M_{R_{k}} L_{k}\right)
$$

Considering ratios of cLFV observables with similar loop dynamics - approximately equal to ratios of the above quantity - , firstly allows to test the SUSY seesaw by checking whether or not its degrees of freedom can accommodate the value of (future) measured quantities. In turn, this may then allow to extract information on the heavy spectrum, $M_{R_{k}}$ (although there is still a dependence on the neutrino mass hierarchy and $U^{\mathrm{MNS}}$ phases). On the other hand, by comparing observables with different loop dynamics but similar flavour structure (e.g., $\ell_{i} \rightarrow 3 \ell_{j}$ and $\ell_{i} \rightarrow \ell_{j} \gamma$ ) one may test new sectors where LFV can be present.

\subsection{Slepton induced cLFV observables}

Slepton flavour mixing can lead to charged lepton flavour violation, manifest in a wide array of observables, at both low-energies (rare processes searched for at high-intensity experiments, such as MEG and BaBar) and high energies (at colliders, above the slepton production threshold). Having one unique source of flavour violation implies that the observables should exhibit some correlation which, as extensively discussed in the literature (see, for example, $[34,36,37,39,41,44]$ ), allows to indirectly probe the high-scale seesaw hypothesis.

At low-energies, virtual sleptons can mediate flavour violating lepton transitions, such as radiative decays, three-body decays and conversion in nuclei. As an example, the radiative decay $\ell_{i} \rightarrow \ell_{j} \gamma$ receives contributions originating from sneutrino-chargino and charged slepton-neutralino loops (see e.g. [68], and references therein). Compared to the SM contributions, which are highly suppressed by powers of $m_{\nu} / M_{W}$, these new contributions can be sizeable provided $m_{\tilde{\ell}_{L}}$ is not too heavy and slepton flavour mixing is large. An analytical understanding of the dependency of $\mathrm{BR}\left(\ell_{i} \rightarrow \ell_{j} \gamma\right)$ on the neutrino Yukawa couplings can be obtained using the LLog approximation. In the limit of very small off-diagonal $\Delta m_{\tilde{L}}^{2}$ entries, one has

$$
\frac{\operatorname{BR}\left(\ell_{i} \rightarrow \ell_{j} \gamma\right)}{\operatorname{BR}\left(\ell_{i} \rightarrow \ell_{j} \nu_{i} \bar{\nu}_{j}\right)} \approx \frac{\alpha^{3} \tan ^{2} \beta}{G_{F}^{2} m_{\mathrm{SUSY}}^{8}}\left|\frac{1}{8 \pi^{2}}\left(m_{\tilde{L}}^{2}+m_{\tilde{\nu}_{R}}^{2}+m_{H_{2}}^{2}+\left|A_{0}^{\nu}\right|^{2}\right)\left(Y^{\nu \dagger} L Y^{\nu}\right)_{i j}\right|^{2} .
$$

The current experimental sensitivity to slepton flavour mixing, i.e. to $\left(\Delta m_{\tilde{L}}^{2}\right)_{i j}$, in other observables such as $\ell_{i} \rightarrow \ell_{j} \bar{\ell}_{k} \ell_{k}$ and $\operatorname{CR}(\mu-e, \mathrm{~N})$, is in general smaller than in $\ell_{i} \rightarrow \ell_{j} \gamma$ [69]. The current 90\% C.L. upper-limits on the cLFV radiative decays are [63, 70]

$$
\operatorname{BR}(\mu \rightarrow e \gamma)<5.7 \times 10^{-13}, \quad \operatorname{BR}(\tau \rightarrow \mu \gamma)<4.4 \times 10^{-8}, \quad \operatorname{BR}(\tau \rightarrow e \gamma)<3.3 \times 10^{-8}
$$


Under the assumption that all off-diagonal entries $\left(Y^{\nu^{\dagger}} L Y^{\nu}\right)_{i j}$ are of the same order of magnitude, the limit on $\operatorname{BR}(\mu \rightarrow e \gamma)$ turns out to be the most constraining. In view of this, and given the very recent experimental MEG bound on the $\operatorname{BR}(\mu \rightarrow e \gamma)$ [63], in the present update we mainly focus on the constraints arising from $\mu \rightarrow e \gamma$.

At high-energy colliders, slepton flavour mixing can be directly probed through $\tilde{\ell}_{L i} \rightarrow$ $\ell_{j} \chi_{1}^{0}$ decays $[32,35,37,39,40,44,45]$. At the LHC sleptons are preferably produced in cascade decays of the form $\tilde{q}_{L} \rightarrow\left\{\chi_{2}^{0}, \chi_{1}^{ \pm}\right\} q^{\prime} \rightarrow \tilde{\ell}_{L}\{\ell, \nu\} q^{\prime}$, provided that these are kinematically allowed. Alternatively, sleptons can also be present in the decay chains of directly produced wino-like $\chi^{0}$ and $\chi^{ \pm}$, which then decay to $\tilde{\ell}_{L}$. If both these modes are not viable, then direct production of slepton pairs through Drell-Yann s-channel $\gamma$ and $Z$ exchanges becomes the only possible slepton production mode. ${ }^{3}$

Despite the missing energy signature which is always present in R-parity conserving SUSY models with a neutral lightest SUSY particle (LSP), strategies to reconstruct sparticle masses have been devised [71-73]. These rely on the assumption that sparticles typically decay to ordinary particles through two body cascade decays, and that the invariant masses that can be formed by combining the momenta of the so-produced SM particles give rise to structures with edges (whose end-points are simple functions of sparticle masses). Assuming that the wino-like $\chi_{2}^{0}$ is heavier than the sleptons, the edge structure of the di-lepton invariant mass distributions $\left(m_{\ell \ell}\right)$ is sensitive to slepton masses due to the decay $\chi_{2}^{0} \rightarrow \tilde{\ell} \ell \rightarrow \ell \ell \chi_{1}^{0}$. An interesting effect of a high-scale SUSY seesaw is the appearance of a third edge in the di-lepton invariant mass distribution, due to an intermediate slepton of a different flavour (i.e., $\tilde{\ell}_{j} \rightarrow \ell_{i} \chi_{1}^{0}$ ), a consequence of slepton flavour mixing (see the detailed analysis of [39]).

\subsection{Flavoured slepton mass differences}

Here we focus on the mass differences between sleptons of different generations (especially the first two, which are dominated by either the left- or right-handed slepton component). In the absence of LFV, flavoured or inter-generational slepton mass differences arise from both $Y^{l}$ and in $A^{l}$ (with $A^{l}=A_{0}^{l} Y^{l}$ at the GUT scale); due to the smallness of the electron and muon Yukawa couplings $\left(Y_{(11,22)}^{l}\right)$, the mass differences between the first two generations is in general well below the $\mathcal{O}(0.1 \%)$ level [39], even in the case of large $\tan \beta$.

Through RG-induced effects involving $Y^{\nu}$ (see the previous subsections), the seesaw introduces additional contributions to slepton mass differences. As is clear from eq. (2.5), these appear in the form of flavour diagonal and non-diagonal contributions to the slepton soft masses. Moreover, and even in the absence of flavour-violating effects (i.e., $i=j$ ), their effects are manifest in an enhancement of the fractional splittings between $m_{\tilde{e}_{L}}$ and $m_{\tilde{\mu}_{L}}$ (no significant effect in the right-handed slepton sector, as LFV in the SUSY seesaw is mostly a left-handed phenomenon), which are defined as

$$
\frac{\Delta m_{\tilde{\ell}}}{m_{\tilde{\ell}}}\left(\tilde{e}_{L}, \tilde{\mu}_{L}\right)=\frac{\left|m_{\tilde{e}_{L}}-m_{\tilde{\mu}_{L}}\right|}{\left\langle m_{\tilde{e}_{L}}, m_{\tilde{\mu}_{L}}\right\rangle} .
$$

\footnotetext{
${ }^{3}$ For a discussion of the prospects of LFV in slepton decays at a future Linear Collider see, for example, [44].
} 
Although in the presence of non-negligible flavour violation the slepton eigenstates correspond to a mixture of the three flavours, we will assume here that the states identified by $\tilde{\ell}_{L}$ are dominated by the corresponding flavour component.

Previous studies [39] (before recent 2013 LHC and MEG results) had suggested that splittings as large as $\sim 10 \%$ could indeed be obtained for sleptons lighter than $1 \mathrm{TeV}$. As mentioned before, we now proceed to re-evaluate these claims, in view of recent MEG bounds and LHC search results.

\section{Numerical results and discussion}

In our numerical analysis we assume a normal hierarchy for the light neutrino spectrum, with non-vanishing $m_{\nu_{1}}$ (which we set $\approx \mathcal{O}\left(10^{-5} \mathrm{eV}\right)$ ). The squared neutrino mass differences, as well as the neutrino mixing angles (for a standard parameterisation of the $U^{\mathrm{MNS}}$ ), are taken in the intervals favoured by current best fits [74]. In our analysis we will assume vanishing CP phases (Dirac and Majorana).

We compute the SUSY spectrum and couplings using the public code SPHENO$3.2 .2[75,76]$, extended by additional routines to fit the high-scale neutrino Yukawa couplings as to yield the observed oscillation data. We require that the lightest Higgs state, $h$, be compatible with recent LHC data on a SM-like scalar boson [59-62]. In addition to having $m_{h}$ in the range $[123 \mathrm{GeV}, 128 \mathrm{GeV}]$, we further require that its couplings are not excluded at 95\% C.L. by current data using HiggsBounds-4.0.0 [77]. Concerning the sparticle spectrum, we have imposed the following (conservative) bounds: all LEP bounds [78] were enforced; the gluino and first two generation squarks are required to be heavier than the upper-limits of [50] (derived in the limit of $m_{\chi_{1}^{0}}=0$ ); bounds on the 3rd generation squark masses [51-54], $m_{\chi_{2}^{0}}, m_{\chi_{1}^{ \pm}}[55,56]$ and $m_{\tilde{\ell}}[57]$ are also imposed (again in simplified models with $m_{\chi_{1}^{0}}=0$ ). If the LSP is charged (solutions which are disfavoured in our phenomenological analysis), we nevertheless require its mass to be above the most constraining lower-limit derived from searches for heavy stable charged particles at the LHC [58]. Finally, the dark-matter relic density is calculated with MICROMEGAs$3.0 .24[79]$.

In our analysis we first begin by considering minimal supergravity (mSUGRA) inspired universality conditions, and afterwards study the impact of relaxing these universality conditions. This serves different purposes. First, to identify the regions of the parameter space offering the most promising prospects for observation of slepton mass differences at forthcoming LHC runs which, besides providing a SM-like Higgs, are compatible with the most recent low energy cLFV and sparticle bounds. Secondly, our aim is to investigate whether the synergy of such mass differences (measurements or upper bounds) and the future results on low energy cLFV could still suggest some hints on the heavy neutrino spectrum (now rendered easier as all light neutrino mixing angles have been measured).

\section{1 mSUGRA-inspired universality}

We first consider an mSUGRA inspired framework, requiring that at $M_{\mathrm{GUT}}$ the SUSY soft-breaking parameters are universal as in cMSSM, and imposing the following relations 
on the additional seesaw soft-breaking terms:

$$
\left(m_{\tilde{\nu}_{R}}^{2}\right)_{i j}=\delta_{i j} m_{0}^{2}, \quad\left(A^{\nu}\right)_{i j}=A_{0}\left(Y^{\nu}\right)_{i j},
$$

where $m_{0}$ and $A_{0}$ are the universal scalar soft-breaking mass and trilinear couplings of the cMSSM. We begin our analysis by revisiting the $m_{0}-M_{1 / 2}$ plane, and evaluate the joint impact of the recent MEG bound on $\mathrm{BR}(\mu \rightarrow e \gamma)$ and of the LHC negative SUSY searches. This further allows to determine the experimentally viable regions offering the best prospects concerning the study of slepton mass splittings.

In figure 1 we present the $m_{0}-M_{1 / 2}$ plane ${ }^{4}$ for different choices of $A_{0}$ and $\tan \beta$, and for distinct seesaw scales. We set the $R$-matrix to $\mathbb{1}$ (see eq. (2.4)), thus working in a conservative scenario where all flavour violation in the lepton sector arises from the $U^{\mathrm{MNS}}$. In each panel, the blue lines denote $\operatorname{BR}(\mu \rightarrow e \gamma)$ isolines: the former MEGA bound, $1.2 \times 10^{-11}$ [81] (lower dashed line), the MEG current bound $5.7 \times 10^{-13}$ [63] (solid line), as well as MEG's expected future sensitivity [82], $6 \times 10^{-14}$ (upper dashed line). In addition, the region delimited by a thick dashed line is excluded by collider bounds [50-58], while the two solid pink lines correspond to having $m_{h} \in[123 \mathrm{GeV}, 128 \mathrm{GeV}]$, in agreement with LHC data [59-62]. Figure 1 clearly manifests the effect of the new $\operatorname{BR}(\mu \rightarrow e \gamma)$ upperlimit, which in all cases amounts to dramatically reducing the previously allowed cMSSM SUSY-seesaw space. By lowering the seesaw scale (i.e., taking smaller values of $M_{R}$, and hence smaller $Y^{\nu}$ ), larger regions of the $m_{0}-M_{1 / 2}$ plane can survive. However, and as we proceed to discuss, this has a direct impact on the prospects for sizeable flavoured slepton mass splittings, as both observables stem from a unique source of LFV - the neutrino Yukawa couplings.

As can be seen in the top left panel of figure 1, the largest fractional mass splittings $\left(\Delta m_{\tilde{\ell}}\left(\tilde{e}_{L}, \tilde{\mu}_{L}\right) / m_{\tilde{\ell}}\right)$ correspond to regions with large $m_{0}$. This can be understood from the fact that (flavour non-universal) RG-driven contributions to the soft masses of left-handed (LH) sleptons are proportional to $m_{\tilde{L}^{2}}^{2}=m_{\tilde{\nu}_{R}}^{2}=m_{H_{2}}^{2}=m_{0}^{2}$ at $M_{\mathrm{GUT}}$ (cf. eq. (2.5)). The predominant effect of a larger $M_{1 / 2}$ translates in the increase of the slepton soft-masses at the SUSY scale from radiative corrections involving EW gauginos. For the case of a comparatively large seesaw scale $\left(M_{R} \sim 10^{15} \mathrm{GeV}\right)$, in association with large Yukawa couplings, $Y^{\nu} \sim \mathcal{O}(1)$, one could expect $\Delta m_{\tilde{\ell}}\left(\tilde{e}_{L}, \tilde{\mu}_{L}\right) / m_{\tilde{\ell}} \approx 2 \%$; however, these regions are associated to $\operatorname{BR}(\mu \rightarrow e \gamma)$ already excluded. Complying with all bounds (accelerator — including Higgs searches -, and low-energy), and further requiring a neutral LSP, ${ }^{5}$ reduces the

\footnotetext{
${ }^{4}$ The recent analysis of [80] suggests that charge and colour breaking minima constraints on the cMSSM parameter space, in particular in the regime of large $\left|A_{0}\right|$, can be more severe than previously thought. A detailed study of whether or not some of points here considered are associated to an unstable desired EWSB minimum lies beyond the scope of our work. However, we expect that the large majority of points compatible with all bounds (including flavour) falls outside the unstability regions identified in [80], where scenarios of large $\left|A_{0}\right|$ are typically associated to very large $M_{1 / 2}$ and/or even larger $m_{0}$.

${ }^{5}$ In our analysis, and other than requiring that the LSP be neutral (typically the lightest neutralino), we do not impose dark matter constraints on the parameter space. For completeness, we notice that in general the relic density $\left(\Omega_{\mathrm{DM}} h^{2}\right)$ is always $\Omega_{\mathrm{DM}} h^{2}>0.13$ [79], as points complying with recent bounds [83, 84] lie below the direct search exclusion line. One can nevertheless consider non-standard cosmological models, where a deviation from standard Big-Bang cosmology allows to reduce the relic density [85, 86], or a very small amount of R-parity violation that would render the LSP unstable.
} 

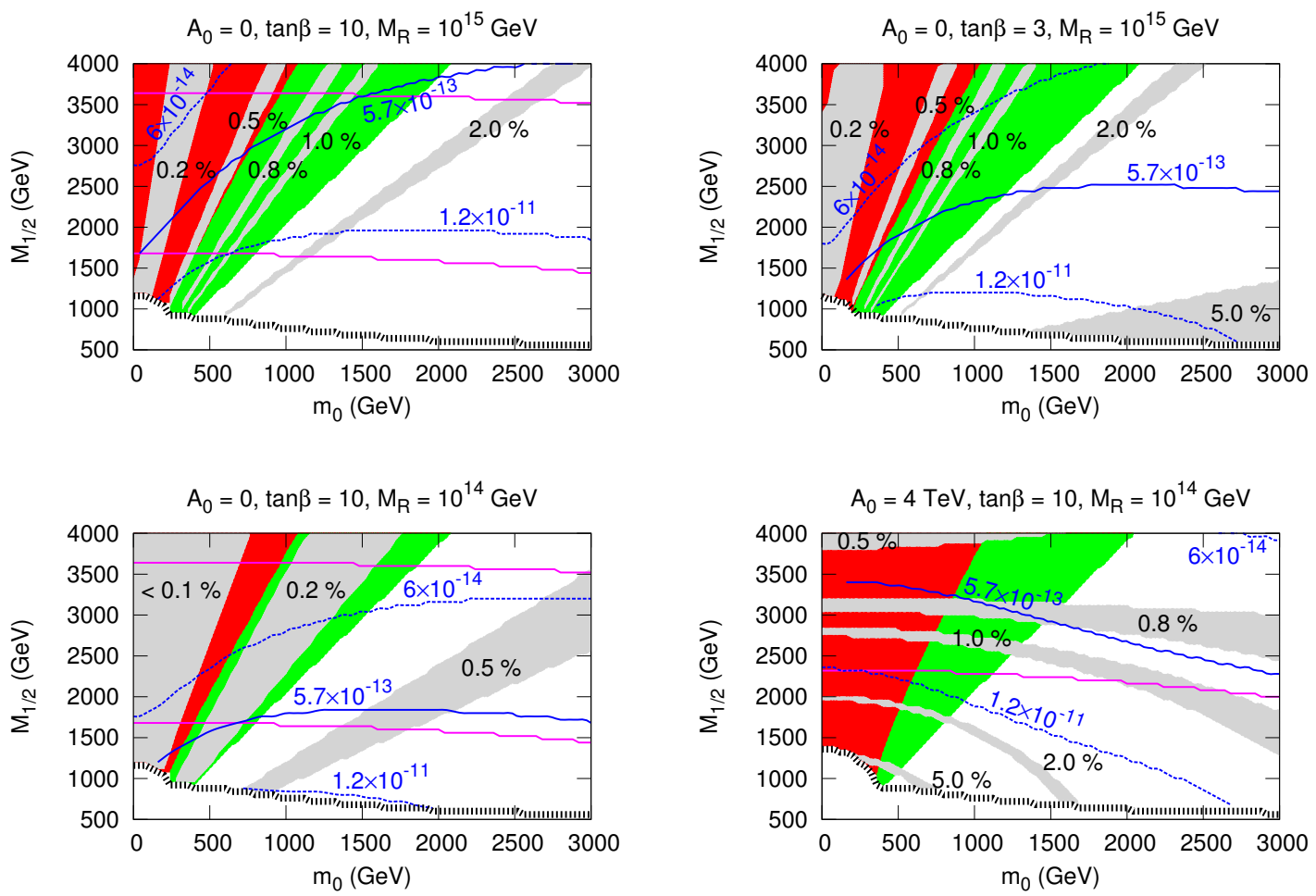

Figure 1. $m_{0}-M_{1 / 2}$ plane for different choices of $A_{0}, \tan \beta$ and seesaw scale $\left(M_{R}\right)$, with different grey regions corresponding to distinct values of $\Delta m_{\tilde{\ell}}\left(\tilde{e}_{L}, \tilde{\mu}_{L}\right) / m_{\tilde{\ell}}$ as indicated. We have taken a degenerate $\mathrm{RH}$ neutrino spectrum and set $R=\mathbb{1}$. The region below the thick (black) dashed curve does not pass the cuts applied on sparticle masses, while the red regions are excluded due to the presence of a charged LSP. Green regions correspond to $\left.m_{\chi_{2}^{0}, \chi_{1}^{ \pm}}\right\rangle\left\langle m_{\tilde{e}_{L}}, m_{\tilde{\mu}_{L}}\right\rangle+10 \mathrm{GeV}$. Different blue curves denote $\mathrm{BR}(\mu \rightarrow e \gamma)$ isolines, while the two solid pink lines enclose the region where $m_{h} \in[123 \mathrm{GeV}, 128 \mathrm{GeV}]$.

$m_{0}-M_{1 / 2}$ plane to a small triangular region, corresponding to $m_{0} \sim 1 \mathrm{TeV}, M_{1 / 2} \sim 3.5 \mathrm{TeV}$, where at most one can expect $\Delta m_{\tilde{\ell}}\left(\tilde{e}_{L}, \tilde{\mu}_{L}\right) / m_{\tilde{\ell}} \sim \mathcal{O}(1 \%)$, typically for $m_{\tilde{\ell}_{L}} \approx 2.5 \mathrm{TeV}$.

As mentioned before, lowering the seesaw scale reduces the amount of RG-induced cLFV. As manifest from the comparison of the left panels of figure 1, the new bound on $\mathrm{BR}(\mu \rightarrow e \gamma)$ can now be accommodated in larger regions of the $m_{0}-M_{1 / 2}$ plane, but slepton mass splittings also diminish, and one has $\Delta m_{\tilde{\ell}}\left(\tilde{e}_{L}, \tilde{\mu}_{L}\right) / m_{\tilde{\ell}} \lesssim 0.5 \%$. Although this will be addressed in more detail in the following section, considering $R \neq \mathbb{1}$ would mostly lead to a displacement of the $\Delta m_{\tilde{\ell}}$ isosurfaces to larger values of $m_{0}$, accompanied by distortions of the $\operatorname{BR}(\mu \rightarrow e \gamma)$ isolines; a hierarchical $\mathrm{RH}$ spectrum (or fixed values of $M_{R_{3}}$ ) would in turn lead to a slight reduction of the associated $\operatorname{BR}(\mu \rightarrow e \gamma)$.

The size (and global shape) of the different regions in the $m_{0}-M_{1 / 2}$ plane also reflects the remaining mSUGRA parameters, $A_{0}$ and $\tan \beta$. The two lower panels of figure 1 reflect the impact of varying the trilinear couplings. For $\left|A_{0}\right| \gg m_{0}$, the $A_{0}^{2}$ contribution outweighs that of $m_{0}^{2}$ to the flavour non-universal radiative corrections (see eq. (2.5)), so that in this case the mass splittings are approximately constant along the $m_{0}$ direction. In the 
regime of large $\left|A_{0}\right|$, one finds that the largest splittings compatible with flavour bounds are $\Delta m_{\tilde{\ell}}\left(\tilde{e}_{L}, \tilde{\mu}_{L}\right) / m_{\tilde{\ell}} \sim 0.8 \%$, for sleptons heavier than several TeV. The effect of varying - in particular, lowering - $\tan \beta$ can be evaluated from the comparison of the two upper panels. Regimes of larger $\tan \beta$ lead to larger SUSY contributions to $\operatorname{BR}(\mu \rightarrow e \gamma)[7,8]$, and compatibility with current bounds strongly constrains the size of the Yukawa couplings, thus reducing the maximal value of the slepton mass differences. Setting $\tan \beta=3$ implies that in a strict cMSSM framework $h$ is too light to be the SM-like Higgs. However, minimally relaxing the universal conditions (in particular concerning the third generation squark masses) to accommodate $m_{h} \sim 125 \mathrm{GeV}$, without affecting considerably the observables being displayed, allows to infer that the low $m_{0}-M_{1 / 2}$ regions that were excluded in the $\tan \beta=10$ case by the upper-limit on $\mu \rightarrow e \gamma$ are now viable. In addition, they exhibit a small enhancement of the mass splittings due to an increase in the strength of $Y^{\nu}\left(\propto 1 / v_{2}\right.$, cf. eq. (2.4)). (Relaxed scenarios, which accommodate $m_{h} \sim 125 \mathrm{GeV}$ with light EW gauginos and sleptons, will be explored in section 3.2.)

To summarise the crucial point of the first part of the analysis, figure 1 clearly reveals how the prospects for probing the cMSSM type-I seesaw have evolved in view of the recent experimental breakthroughs. While in a first analysis ${ }^{6}$ (pre-LHC) [39] one could have $\Delta m_{\tilde{\ell}}\left(\tilde{e}_{L}, \tilde{\mu}_{L}\right) / m_{\tilde{\ell}} \sim 5 \%$ for $m_{\tilde{\ell}} \sim 500 \mathrm{GeV}$, one is now confronted to a very different situation: at most one can expect $\Delta m_{\tilde{\ell}}\left(\tilde{e}_{L}, \tilde{\mu}_{L}\right) / m_{\tilde{\ell}} \sim 1 \%$, and only in a somewhat fine-tuned region of the $m_{0}-M_{1 / 2}$ plane, always in association with considerably heavy sleptons $(\sim 2.5 \mathrm{TeV})$. Whether or not the LHC will be able to reconstruct such tiny mass differences lies beyond the scope of the present analysis.

As mentioned in section 2.2, at the LHC sleptons are preferably produced in $\tilde{q}_{L} \rightarrow$ $\left\{\chi_{2}^{0}, \chi_{1}^{ \pm}\right\} \rightarrow \tilde{\ell}_{L}$ decays (if kinematically allowed). In all panels of figure 1, green surfaces correspond to regions where $\tilde{\ell}_{L}$ can be produced from $\chi_{2}^{0}$ decays, accompanied by the emission of a hard lepton. We will subsequently explore these regions in greater detail. In particular, and instead of considering a degenerate $\mathrm{RH}$ neutrino spectrum, we now consider a hierarchical one: we fix $M_{R_{1}}=10^{10} \mathrm{GeV}, M_{R_{2}}=10^{11} \mathrm{GeV}$ with $M_{R_{3}} \in$ $\left\{10^{12}, 10^{13}, 10^{14}, 10^{15}\right\} \mathrm{GeV}$. We again set $R=\mathbb{1}$. For each point we then perform a random scan in $m_{0}, M_{1 / 2}$ and $A_{0}$, which are chosen from the following wide ranges

$$
m_{0} \in[0,3] \mathrm{TeV}, M_{1 / 2} \in[0,10] \mathrm{TeV}, A_{0} \in[-4,4] \mathrm{TeV} .
$$

The low-energy spectrum is subject to all the aforementioned cuts on sparticle (and lightest Higgs) masses. One further requires that the spectrum obeys $m_{\chi_{2}^{0}}>\left\langle m_{\tilde{e}_{L}}, m_{\tilde{\mu}_{L}}\right\rangle+10 \mathrm{GeV}$, and that $\chi_{1}^{0}$ is the LSP. The results are collected in figure 2, where we display the slepton mass splittings versus the average slepton mass for the first two generations.

Each of the panels of figure 2 comprises four "boomerang-shaped" regions, corresponding to the different choices of $M_{R_{3}}$ (increasing from left to right). Within each individual

\footnotetext{
${ }^{6}$ In a previous exploratory study [39], a regime of very small $\theta_{13}$ (prior to its experimental measurement) had been considered. For larger $\theta_{13}$, the largest slepton mass splittings compatible with the same set of flavour bounds are smaller than those derived for small $\theta_{13}$. In fact, in the case $R \approx \mathbb{1}$ and for $M_{R_{3}} \gg M_{R_{1,2}}$, smaller values of $\theta_{13}$ allow for a larger overall contribution to $\Delta m_{\tilde{\ell}}\left(\tilde{e}_{L}, \tilde{\mu}_{L}\right) / m_{\tilde{\ell}}$ (which can also proceed from $\tilde{\tau}-\tilde{\mu}$ mixing, less experimentally constrained).
} 

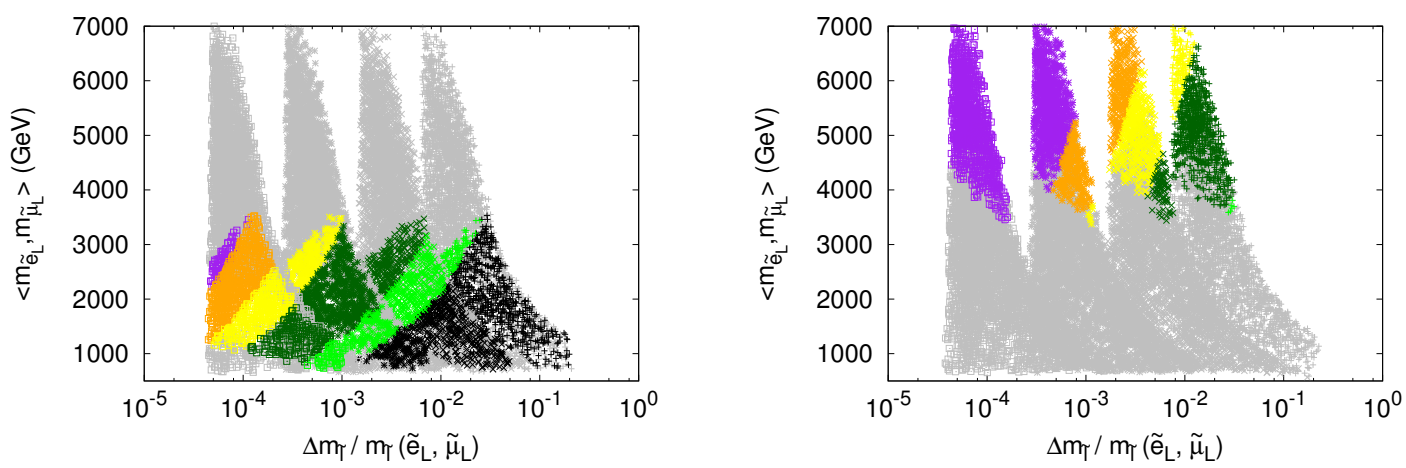

Figure 2. Slepton mass splittings versus the average slepton mass for the first two generations of mostly LH sleptons, in the cMSSM type-I seesaw. On the left we set $\tan \beta=10$, while on the right $\tan \beta=3$. We have taken $R=\mathbb{1}$ and a hierarchical RH neutrino spectrum with $M_{R_{1}}=10^{10} \mathrm{GeV}$, $M_{R_{2}}=10^{11} \mathrm{GeV}$ and $M_{R_{3}}=\left\{10^{12}, 10^{13}, 10^{14}, 10^{15}\right\} \mathrm{GeV}$ (corresponding to the four regions along the $\Delta m_{\tilde{\ell}}\left(\tilde{e}_{L}, \tilde{\mu}_{L}\right) / m_{\tilde{\ell}}$ axis). Grey points have $m_{h}$ outside the preferred interval; purple, orange, yellow, dark-green, light-green and black regions correspond to $\operatorname{BR}(\mu \rightarrow e \gamma)$ in the ranges $<10^{-17}$, $\left[10^{-17}, 10^{-16}\right],\left[10^{-16}, 10^{-15}\right],\left[10^{-15}, 6 \times 10^{-14}\right],\left[6 \times 10^{-14}, 5.7 \times 10^{-13}\right]$ and $>5.7 \times 10^{-13}$, respectively.

region, the upwards (and left-most) part corresponds to regimes of small $\left|A_{0}\right|$ (compared to $m_{0}$ and $M_{1 / 2}$ ), while the right-most extremity is associated with larger $\left|A_{0}\right|$. As it is clear from the left panel (where $\tan \beta=10$ ), any splittings $\gtrsim \mathcal{O}(1 \%)$, compatible with current lepton flavour bounds, are associated to heavy sleptons, $m_{\tilde{\ell}} \sim 2 \mathrm{TeV}$. In agreement to what could be expected from the discussion ${ }^{7}$ of figure 1 , these large splittings can either occur for $M_{R_{3}} \approx 10^{15} \mathrm{GeV}$ in the small $\left|A_{0}\right|$ regime or $M_{R_{3}} \approx 10^{14} \mathrm{GeV}$ for large $\left|A_{0}\right|$; we find it worth emphasising that these sizeable splittings, which have an associated $\operatorname{BR}(\mu \rightarrow e \gamma)$ in the range of MEG's expected future sensitivity (light-green points) have a spectrum compatible with $m_{h} \sim 125 \mathrm{GeV}$. It is nevertheless possible to have $\Delta m_{\tilde{\ell}}\left(\tilde{e}_{L}, \tilde{\mu}_{L}\right) / m_{\tilde{\ell}} \gtrsim \mathcal{O}(0.1 \%)$, compatible with current flavour bounds and within MEG reach, for lighter sleptons.

For comparison, on the right panel of figure 2 we display an analogous study for $\tan \beta=$ 3. As previously discussed, in such a regime, complying with $m_{h}$ bounds requires very large values of $m_{0}$ and $M_{1 / 2}$ (in a strictly constrained MSSM framework), thus leading to extremely heavy sleptons (and gauginos), $m_{\tilde{\ell}_{L}} \gtrsim 3.5 \mathrm{TeV}$. In turn, this precludes the possibility of observing cLFV transitions at MEG (essentially for any value of the mass splittings).

Figure 2 clearly suggests that scenarios with sizeable $\Delta m_{\tilde{\ell}}\left(\tilde{e}_{L}, \tilde{\mu}_{L}\right) / m_{\tilde{\ell}}$, in association to a slepton spectrum sufficiently light to be abundantly produced at the LHC, and with viable $\operatorname{BR}(\mu \rightarrow e \gamma)$ are excluded, since in a constrained framework as the cMSSM, such regimes are not compatible with $m_{h}$. In the following, we consider the impact of relaxing the strict universality of the SUSY soft-breaking terms regarding slepton mass splittings.

\footnotetext{
${ }^{7}$ We notice that a degenerate RH neutrino spectrum was considered in the analysis of figure 1. For fixed values of $M_{R_{3}}$, a hierarchical $\mathrm{RH}$ spectrum leads to a slight reduction of the associated $\mathrm{BR}(\mu \rightarrow e \gamma)-$ by around a factor $2-$, hence rendering viable part of the $1 \%$ band in the bottom-right panel of figure 1.
} 


\subsection{Beyond mSUGRA-inspired universal conditions}

We now consider a modified SUSY seesaw scheme in which one breaks strict universality for squark, slepton and Higgs soft-breaking terms at the GUT scale (but still preserving flavour universality). Moreover, soft breaking gluino and EW gaugino masses are also taken to be independent. This results in the following relations at $M_{\mathrm{GUT}}$, yielding 7 free parameters in addition to $\tan \beta$ and $\operatorname{sign}(\mu)$ :

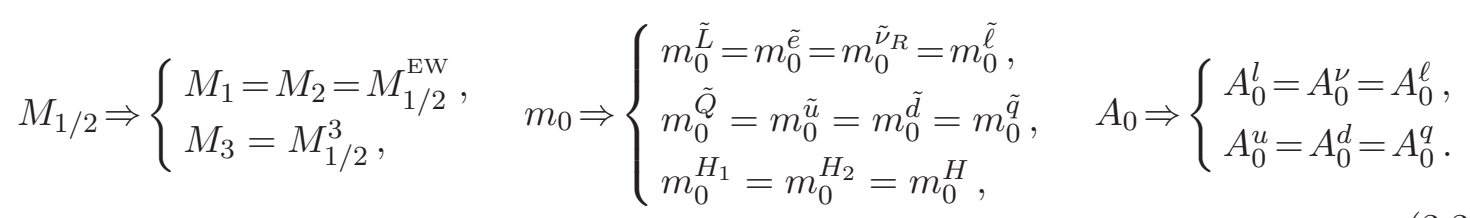

For each of the two considered regimes for $\tan \beta$, we focus on the following specific choices for $M_{1 / 2}^{3}, m_{0}^{\tilde{q}}$ and $A_{0}^{q}$, which lead to $m_{h} \sim 125 \mathrm{GeV}$ (enhanced by radiative corrections involving heavy stops and/or large stop mixing),

$$
\begin{array}{llll}
\tan \beta=10: & M_{1 / 2}^{3}=1.1 \mathrm{TeV}, & m_{0}^{\tilde{q}}=1.5 \mathrm{TeV}, & A_{0}^{q}=-4 \mathrm{TeV} \\
\tan \beta=3: & M_{1 / 2}^{3}=4.7 \mathrm{TeV}, & m_{0}^{\tilde{q}}=4.5 \mathrm{TeV}, & A_{0}^{q}=-15 \mathrm{TeV} ;
\end{array}
$$

and, similar to what was done for figure 2, we conduct a random scan of the remaining parameters, which were varied in the following chosen intervals:

$$
M_{1 / 2}^{\mathrm{EW}} \in[0,5] \mathrm{TeV}, m_{0}^{\tilde{\ell}} \in[0,3] \mathrm{TeV}, m_{0}^{H} \in[0,3] \mathrm{TeV}, A_{0}^{\ell} \in[-5,5] \mathrm{TeV} .
$$

The results are collected in figure 3 , the panels corresponding to $\tan \beta=10$ (left) and $\tan \beta=3$ (right). All points displayed are in agreement with LHC bounds on sparticle masses and on a SM-like Higgs mass. Moreover, the spectrum always fulfils $m_{\chi_{2}^{0}}>\left\langle m_{\tilde{e}_{L}}, m_{\tilde{\mu}_{L}}\right\rangle+10 \mathrm{GeV}$, with a $\chi_{1}^{0} \mathrm{LSP}$.

As it is manifest from figure 3, the most interesting consequence of relaxing the strict cMSSM universality conditions concerns the possibility of having considerably lighter sleptons in association with sizeable mass splittings and still compatible with flavour bounds. This is particularly striking in the case of $\tan \beta=3$ (right panel), where one can verify that sleptons as light as $\sim 800 \mathrm{GeV}(1.6 \mathrm{TeV})$ can be associated to $\mathcal{O}(0.1 \%)$ $(\mathcal{O}(1 \%))$ splittings, in agreement with all imposed constraints. For $\tan \beta=10$ (displayed on the left panel), the impact of deviating from a strict cMSSM framework is somewhat less pronounced: one again finds that $\mathcal{O}(0.1 \%)$ slepton mass splittings are attainable for $m_{\tilde{\ell}} \gtrsim 0.9 \mathrm{TeV}$. Larger values of $\Delta m_{\tilde{\ell}}\left(\tilde{e}_{L}, \tilde{\mu}_{L}\right) / m_{\tilde{\ell}}$ remain difficult to obtain with the boundary conditions of eq. (3.4).

A very interesting feature of this relaxed framework, especially for the $\tan \beta=10$ case, is that the spectrum now allows for an efficient LSP density depletion (via $\chi_{1}^{0}-\tilde{t}_{1}$ co-annihilation). This is highlighted by the red points in the left panel of figure 3 .

Concerning the prospects of a potential future observation of a $\mu \rightarrow e \gamma$ decay at MEG, in the case of $\tan \beta=10$, and for sleptons lighter than $1.2 \mathrm{TeV}, \Delta m_{\tilde{\ell}}\left(\tilde{e}_{L}, \tilde{\mu}_{L}\right) / m_{\tilde{\ell}} \gtrsim \mathcal{O}(0.1 \%)$ are associated to $\operatorname{BR}(\mu \rightarrow e \gamma)$ within MEG's expected future sensitivity. For $\tan \beta=3$, 

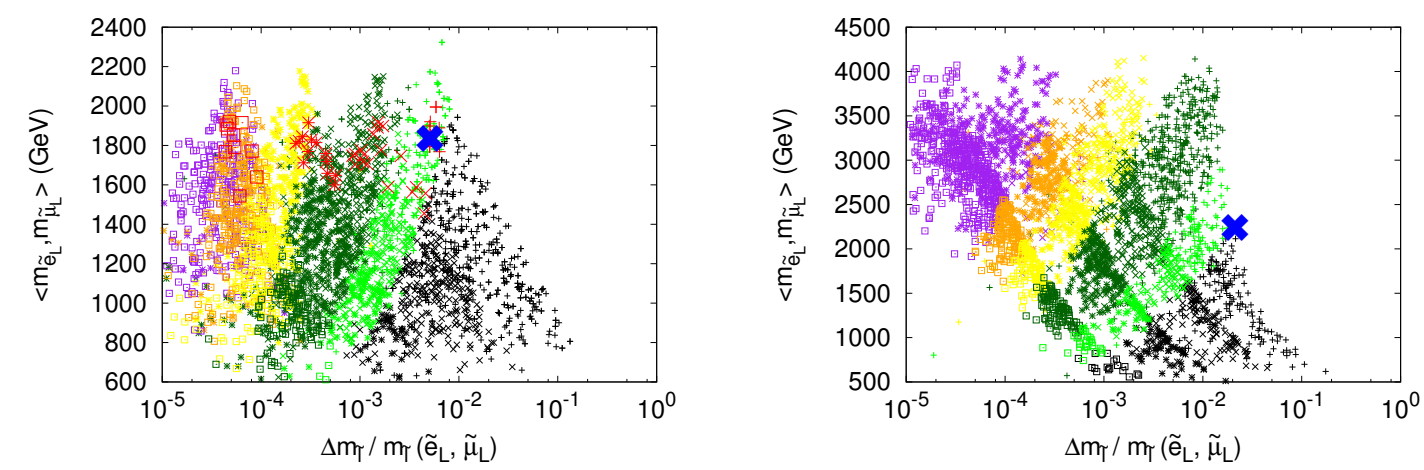

Figure 3. Mass splittings versus average mass for the first two generations of mostly LH sleptons, for the scenario of eq. (3.3). The underlying scan is described in the text, with $\tan \beta=10$ (3) in the left (right) panel. We have taken $R=\mathbb{1}$, and a hierarchical RH neutrino spectrum with $M_{R_{1}}=10^{10} \mathrm{GeV}, M_{R_{2}}=10^{11} \mathrm{GeV}$ and $M_{R_{3}}=\left\{10^{12}, 10^{13}, 10^{14}, 10^{15}\right\} \mathrm{GeV}$. Colour code as in figure 2; in addition, we denote in red the points exhibiting a DM relic density $\Omega_{\mathrm{DM}} h^{2}<0.13$. Superimposed blue "crosses" correspond to the sample points of table 1.

splittings $\gtrsim \mathcal{O}(1 \%)(\mathcal{O}(0.1 \%))$, with sleptons lighter than $\sim 2.7 \mathrm{TeV}(1.3 \mathrm{TeV})$, would also yield $\operatorname{BR}(\mu \rightarrow e \gamma)$ values within MEG reach.

As compared to figure 2, where one could still identify four independent "boomerang" shapes (especially for heavy sleptons), here only four small crests can be distinguished (again for the heavier slepton regimes). Due to having uncorrelated $m_{0}^{\tilde{q}}$ and $m_{0}^{H}$ at the GUT scale, and having taken heavy stops (with large $A_{0}^{u}$ ), $m_{H_{2}}^{2}$ can now run to negative values above the seesaw scale, thus potentially cancelling the contribution of $m_{\tilde{\nu}_{R}}^{2}, m_{\tilde{L}}^{2}$ and $\left|A_{0}^{\nu}\right|^{2}$ to the flavour violating RG-induced effects (cf. eq. (2.5)). Potential cancellations within the flavour-violating soft-breaking terms were pointed out in [66] for the case of non-universal Higgs masses (where possible negative values of $m_{\mathrm{H}_{2}}^{2}$ at the GUT scale were considered). Finally, we notice that points in the left (right) panel have an average squark mass for the first two LH generations $m_{\tilde{q}_{L}} \sim 3(9) \mathrm{TeV}$. Although this formally means that all points displayed in figure 3 would have the $\tilde{q}_{L} \rightarrow \chi_{2}^{0} \rightarrow \tilde{\ell}_{L}$ cascade open, in the general case the most likely slepton production mode remains via direct gaugino production (due to the very heavy squark spectrum).

In what follows we evaluate to which extent the correlations between low- and highenergy cLFV observables can still provide potential probes of a type-I SUSY seesaw, illustrating the results for two individual cases, singled out from the panels of figure 3, where they have been depicted using blue "crosses".

Point A (left panel $-\tan \beta=10$ ) exhibits the largest splittings for a light slepton spectrum, and has $\Omega_{\mathrm{DM}} h^{2}$ within the $1 \sigma$ interval of Planck [83]. Point B (right panel $\tan \beta=3$ ) also corresponds to a choice illustrating the largest splittings still compatible with current flavour bounds. In table 1 we display the SUSY soft breaking terms (in addition to the fixed input parameters of eq. (3.4)), and a sample of the SUSY spectrum corresponding to $M_{R_{3}}=10^{15} \mathrm{GeV}$. Strictly for illustrative purposes, and using Prospino$2.1[87,88]$, we also provide an estimation of the LH slepton production cross-sections for 


\begin{tabular}{|c|c|c|}
\hline & $\mathrm{A}$ & $\mathrm{B}$ \\
\hline$\left\langle m_{\tilde{e}_{L}}, m_{\tilde{\mu}_{L}}\right\rangle$ & 1835 & 2240 \\
\hline$\frac{\Delta m_{\tilde{\ell}}}{m_{\tilde{\ell}}}\left(\tilde{e}_{L}, \tilde{\mu}_{L}\right)$ & $0.5 \%$ & $2.1 \%$ \\
\hline$m_{\chi_{2}^{0}, \chi_{1}^{ \pm}}$ & 1936 & 2349 \\
\hline$m_{\chi_{1}^{0}}$ & 1043 & 1264 \\
\hline$m_{\tilde{t}_{1}}$ & 1084 & 4825 \\
\hline$\left\langle m_{\tilde{q}_{L}}\right\rangle$ & 2916 & 9059 \\
\hline$m_{h}$ & 125.4 & 125.3 \\
\hline
\end{tabular}

\begin{tabular}{|l|c|c|}
\hline & $\mathrm{A}$ & $\mathrm{B}$ \\
\hline$\sigma_{\tilde{\ell}}^{\operatorname{prod}\left(\chi_{2}^{0}, \chi_{1}^{ \pm}\right)}$ & $6.1 \times 10^{-4}$ & $7.6 \times 10^{-4}$ \\
\hline$\sigma_{\tilde{\ell}}^{\operatorname{prod}\left(\chi_{2}^{0} \text { only }\right)}$ & $2.1 \times 10^{-4}$ & $2.6 \times 10^{-4}$ \\
\hline$\sigma_{\tilde{\ell}}^{\operatorname{prod}\left(\tilde{q}_{L} ; \text { via } \chi_{2}^{0}, \chi_{1}^{ \pm}\right)}$ & $1.9 \times 10^{-2}$ & - \\
\hline$\sigma_{\tilde{\ell}}^{\text {prod }\left(\tilde{q}_{L} ; \text { via } \chi_{2}^{0} \text { only }\right)}$ & $6.4 \times 10^{-3}$ & - \\
\hline
\end{tabular}

Table 1. Points A and B: SUSY soft breaking input parameters, sample of the SUSY spectrum and slepton mass splittings (in the case of $M_{R_{3}}=10^{15} \mathrm{GeV}$ ), and LH slepton production cross-sections for $\sqrt{s}=14 \mathrm{TeV}$ (masses in $\mathrm{GeV}$ and $\sigma$ in fb).

$\sqrt{s}=14 \mathrm{TeV}$, from the decay of directly produced neutral and charged winos $\left(\chi_{2}^{0}, \chi_{1}^{ \pm}\right)$, as well as from the decay chains of directly produced squarks and gluinos. (Notice that Point $\mathrm{A}$ is a concrete example of a case where slepton production via squark production is more favourable than via direct gaugino production.)

Although the production cross-sections for these points suggest that observation of a significant number of events might be challenging, it is important to stress that a minor modification of the spectra (in particular, breaking the universality of the third generation of squarks) could easily lead to scenarios where as much as 300 events can be indeed achieved, for an integrated luminosity around $3000 \mathrm{fb}^{-1}$.

In figure 4 we display $\operatorname{BR}(\mu \rightarrow e \gamma)$ as a function of $\Delta m_{\tilde{\ell}}\left(\tilde{e}_{L}, \tilde{\mu}_{L}\right) / m_{\tilde{\ell}}$ for points $\mathrm{A}$ (left panel) and B (right panel), considering different regimes of $M_{R_{3}}$ (for fixed values of $\left.M_{R_{1,2}}\right)$. On the secondary y-axis we present the corresponding value of $\mathrm{CR}(\mu-e$, $\mathrm{Ti})$, estimated assuming the hypothesis of $\gamma$-penguin domination, valid for the scenarios here considered (see, for example [29,69]), and which predicts $\mathrm{CR}(\mu-e, \mathrm{Ti}) \approx 5 \times 10^{-3}$ $\mathrm{BR}(\mu \rightarrow e \gamma)$ [13]. A horizontal full (dashed) line corresponds to MEG's current bound (expected future sensitivity), while a cyan dashed line corresponds to PRISM/PRIME proposed sensitivity, ${ }^{8} \mathrm{CR}(\mu-e, \mathrm{Ti}) \sim 10^{-18}$ [91]. On the upper two plots, we have again taken the conservative - yet simple - limit of $R=\mathbb{1}$. On the lower plots of figure 4 , we consider

\footnotetext{
${ }^{8}$ Other proposals for high-sensitivity $\mu-e$ conversion searches (Aluminium nuclei) include Mu2e [89] and COMET [90].
} 

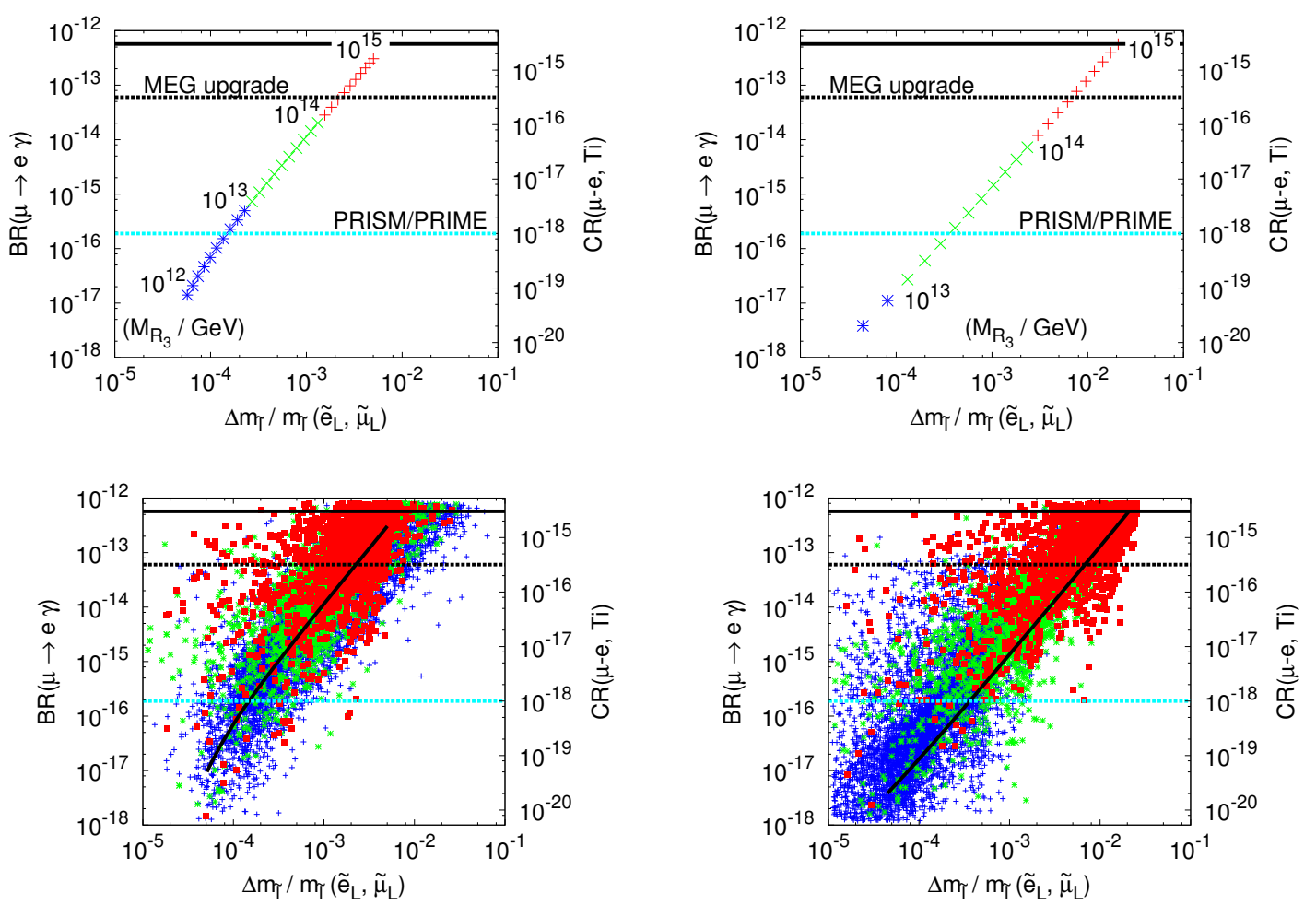

Figure 4. Mass splittings versus $\mathrm{BR}(\mu \rightarrow e \gamma)$ for points A (left) and B (right) — see table 1, displaying complementary information on $\mathrm{CR}(\mu-e, \mathrm{Ti})$ on the secondary y-axis. Full (dashed) horizontal lines denote current bounds (future sensitivities). We have taken a hierarchical RH neutrino spectrum with $M_{R_{1}}=10^{10} \mathrm{GeV}, M_{R_{2}}=10^{11} \mathrm{GeV}$. The colour scheme denotes different intervals of $M_{R_{3}}$ : in the upper panels it is varied in the range $\left[10^{12}, 10^{15}\right] \mathrm{GeV}$, while in the lower panels we considered $10^{13,14,15} \mathrm{GeV}$ (blue, green and red points, respectively). On the upper panels, we set $R=\mathbb{1}$, while in the lower ones the $R$-matrix was randomly varied in the intervals $\left|\theta_{i}\right| \lesssim \pi$ and $-\pi \lesssim \arg \left(\theta_{i}\right) \lesssim \pi$. The slanted full black lines on the lower panels correspond to the limit $R=\mathbb{1}$ depicted in the upper panels.

the more general case where there are additional mixings involving the $\mathrm{RH}$ neutrinos, conducting a broad scan over the complex $R$-matrix angles, $\theta_{i}$. This allows a global overview of the contributions to the different cLFV observables (albeit for fixed SUSY points).

The information contained in the different panels of figure 4 provides a comprehensive summary of the discussion we have conducted so far. Taking into account the additional degrees of freedom in the $\mathrm{RH}$ neutrino sector (encoded in the $R$-matrix complex angles) allows to have mass splittings as large as $5 \%$, still in agreement with low-energy flavour bounds, and even for a comparatively low seesaw scale, $\mathcal{O}\left(10^{13} \mathrm{GeV}\right)$, as made clear from the lower panels. Strong deviations from the simplistic $R=\mathbb{1}$ case may also point in the direction of particular flavour models, as recently explored in [45].

From the interplay of the different observables, and depending on the outcome of the distinct low- and high-energy experiments, many conclusions can be drawn with respect to the viability of a type-I SUSY seesaw as the underlying mechanism.

Under the hypothesis that the slepton mass scale will have been determined (and the sparticle spectrum interpreted in terms of a high-scale SUSY model), a joint study of 
the low- and high-energy cLFV observables can offer important insight into the seesaw dynamics. Let us first consider the simple $R=\mathbb{1}$ case: for scenario $\mathrm{A}$, any measurement of $\Delta m_{\tilde{\ell}}\left(\tilde{e}_{L}, \tilde{\mu}_{L}\right) / m_{\tilde{\ell}} \gtrsim 0.1 \%$ would entail $\mathrm{BR}(\mu \rightarrow e \gamma)$ within MEG reach (and vice-versa), further suggesting a seesaw scale of the order of $10^{14} \mathrm{GeV}$. For reconstructed SUSY models of lower $\tan \beta$, the result in the upper right panel would allow for similar conclusions: splittings of $\mathcal{O}(1 \%)(\mathcal{O}(0.1 \%))$ should be accompanied by the observation of $\mu \rightarrow e \gamma$ decay at MEG ( $\mu-e$ conversion at PRISM/PRIME), hinting towards a seesaw scale of the order of $5 \times 10^{14} \mathrm{GeV}\left(10^{14} \mathrm{GeV}\right)$. Conversely, the isolated manifestation of either low- or high-scale cLFV, e.g. $\Delta m_{\tilde{\ell}}\left(\tilde{e}_{L}, \tilde{\mu}_{L}\right) / m_{\tilde{\ell}} \gtrsim \mathcal{O}(5 \%)$ without any $\mu \rightarrow e \gamma$ or $\mu-e$ signal, would strongly suggest that sources of LFV, other than — or in addition to - the SUSY seesaw are present.

Analogous, but stronger conclusions can be drawn from the inspection of the scans corresponding to $R \neq \mathbb{1}$ case: although the clear dependence on the seesaw scale (present for $R=\mathbb{1}$ ) becomes diluted due to the additional contributions from the mixings involving $\mathrm{RH}$ neutrinos, the correlation between the observables still allows to indirectly test the SUSY seesaw. Again, any measurement $\Delta m_{\tilde{\ell}}\left(\tilde{e}_{L}, \tilde{\mu}_{L}\right) / m_{\tilde{\ell}} \gtrsim \mathcal{O}(1 \%)$ must be accompanied by observation of $\mu \rightarrow e \gamma$ decay at MEG so to substantiate the SUSY seesaw hypothesis; on the other hand $\Delta m_{\tilde{\ell}}\left(\tilde{e}_{L}, \tilde{\mu}_{L}\right) / m_{\tilde{\ell}} \gtrsim \mathcal{O}(1 \%)$ without any $\mu \rightarrow e \gamma$ or $\mu-e$ signal would strongly disfavour the underlying hypothesis.

\section{Conclusions}

In this study we have revisited the impact of a type-I SUSY seesaw concerning LFV following the recent MEG bound on $\operatorname{BR}(\mu \rightarrow e \gamma$ ), LHC data (discovery of a Higgs-like boson and negative SUSY searches), and the measurement of $\theta_{13}$, updating the results obtained in [39]. The aim of our work was to discuss whether current cLFV results and SUSY searches still render viable the observation of slepton mass differences at the LHC, and if the interplay of the latter observables with low-energy cLFV bounds could still shed some light on the high-energy seesaw parameters. Our analysis was based in the hypothesis that all flavour violation in the lepton sector is due to the neutrino Yukawa couplings; we thus embed the type-I seesaw in constrained and semi-constrained SUSY breaking scenarios.

Due to the new $\operatorname{BR}(\mu \rightarrow e \gamma)$ bound, in association with the measured "large" value of $\theta_{13}$, we find that in general slepton mass splittings tend to be very small, unless the slepton spectra is considerably heavy. This implies that for the type-I SUSY seesaw the observation of cLFV at high-energies will be clearly much more challenging than the low-energy, high-intensity studies.

Regarding the embedding of the type-I seesaw into constrained SUSY models such as the cMSSM, we have verified that recent LHC data (in particular the measurement of $\left.m_{h}\right)$ precludes the possibility of simultaneously having $\mathrm{BR}(\mu \rightarrow e \gamma)$ within MEG reach and sizeable slepton mass differences associated with a slepton spectrum sufficiently light to be produced.

On the other hand, relaxing the strict universality of SUSY soft-breaking terms allows to circumvent some of the strongest LHC bounds, especially on $m_{h}$, and opens the door to non-negligible slepton mass splittings (for a comparatively light slepton 
spectrum), with associated $\mu \rightarrow e \gamma$ rates (as well as $\mathrm{CR}(\mu-e)$ ) within experimental reach. Although dependent on the SUSY regime (e.g., on $\tan \beta$ ), one can still find $\Delta m_{\tilde{\ell}}\left(\tilde{e}_{L}, \tilde{\mu}_{L}\right) / m_{\tilde{\ell}} \sim 0.1 \%-1 \%$, for $m_{\tilde{\ell}}$ ranging from $800 \mathrm{GeV}$ to $1.6 \mathrm{TeV}$.

We have studied in detail the impact of the different seesaw parameters for representative points in SUSY space. The results of this comprehensive analysis were presented in figure 4 . As we have shown, one can have mass splittings as large as 5\%, still in agreement with low-energy flavour bounds, even for a comparatively low seesaw scale, $\mathcal{O}\left(10^{13} \mathrm{GeV}\right)$. In these scenarios, one can still use the correlation of high- and low-energy cLFV observables to probe the SUSY seesaw, and we provided some illustrative examples of this interplay.

In summary, our analysis shows that in the case of semi-constrained (flavour universal) SUSY models, the reconstruction of the slepton mass scale and slepton mass splittings, in synergy with the measurement of low-energy cLFV observables, still remains a potential probe to test (strengthen or disfavour) the high-scale type-I SUSY seesaw.

\section{Acknowledgments}

We are indebted to A. Abada and J. C. Romão for many valuable exchanges and suggestions. The work of A. J. R. F. has been supported by Fundação para a Ciência e a Tecnologia through the fellowship SFRH/BD/64666/2009. A. J. R. F. acknowledges the financial support from the EU Network grant UNILHC PITN-GA-2009-237920 and from Fundação para a Ciência e a Tecnologia grants CFTP-FCT UNIT 777, CERN/FP/83503/2008 and PTDC/FIS/102120/2008. We also acknowledge partial support from the European Union FP7 ITN INVISIBLES (Marie Curie Actions, PITN-GA-2011-289442).

Open Access. This article is distributed under the terms of the Creative Commons Attribution License (CC-BY 4.0), which permits any use, distribution and reproduction in any medium, provided the original author(s) and source are credited.

\section{References}

[1] F. Borzumati and A. Masiero, Large muon and electron number violations in supergravity theories, Phys. Rev. Lett. 57 (1986) 961 [INSPIRE].

[2] P. Minkowski, $\mu \rightarrow e \gamma$ at a rate of one out of 1-billion muon decays?, Phys. Lett. B 67 (1977) 421 [INSPIRE].

[3] M. Gell-Mann, P. Ramond and R. Slansky, Complex spinors and unified theories, in Supergravity, P. Van. Nieuwenhuizen and D. Z. Freedman eds., North-Holland, Amsterdam The Netherlands (1979), pg. 315 [PRINT-80-0576] [Conf. Proc. C 790927 (1979) 315] [arXiv: 1306.4669] [INSPIRE].

[4] T. Yanagida, Horizontal symmetry and masses of neutrinos, in Proceedings of the Workshop on the Unified Theory and the Baryon Number in the Universe, O. Sawada and A. Sugamoto eds., KEK, Tsukuba Japan (1979), pg. 95 [Conf. Proc. C 7902131 (1979) 95] [InSPIRE].

[5] S.L. Glashow, The future of elementary particle physics, in Quarks and leptons, M. Lévy et al. eds., Plenum Press, New York U.S.A. (1980), pg.687 [NATO Adv. Study Inst. Ser. B Phys. 59 (1980) 687] [INSPIRE]. 
[6] R.N. Mohapatra and G. Senjanović, Neutrino mass and spontaneous parity violation, Phys. Rev. Lett. 44 (1980) 912 [INSPIRE].

[7] J. Hisano, T. Moroi, K. Tobe and M. Yamaguchi, Lepton flavor violation via right-handed neutrino Yukawa couplings in supersymmetric Standard Model, Phys. Rev. D 53 (1996) 2442 [hep-ph/9510309] [INSPIRE].

[8] J. Hisano, T. Moroi, K. Tobe, M. Yamaguchi and T. Yanagida, Lepton flavor violation in the supersymmetric Standard Model with seesaw induced neutrino masses, Phys. Lett. B 357 (1995) 579 [hep-ph/9501407] [INSPIRE].

[9] J. Hisano and D. Nomura, Solar and atmospheric neutrino oscillations and lepton flavor violation in supersymmetric models with the right-handed neutrinos, Phys. Rev. D 59 (1999) 116005 [hep-ph/9810479] [inSPIRE].

[10] W. Buchmüller, D. Delepine and F. Vissani, Neutrino mixing and the pattern of supersymmetry breaking, Phys. Lett. B 459 (1999) 171 [hep-ph/9904219] [INSPIRE].

[11] Y. Kuno and Y. Okada, Muon decay and physics beyond the Standard Model, Rev. Mod. Phys. 73 (2001) 151 [hep-ph/9909265] [INSPIRE].

[12] J.R. Ellis, M. Gomez, G. Leontaris, S. Lola and D.V. Nanopoulos, Charged lepton flavor violation in the light of the Super-Kamiokande data, Eur. Phys. J. C 14 (2000) 319 [hep-ph/9911459] [INSPIRE].

[13] J. Hisano and K. Tobe, Neutrino masses, muon g-2 and lepton flavor violation in the supersymmetric seesaw model, Phys. Lett. B 510 (2001) 197 [hep-ph/0102315] [INSPIRE].

[14] J. Casas and A. Ibarra, Oscillating neutrinos and $\mu \rightarrow e, \gamma$, Nucl. Phys. B 618 (2001) 171 [hep-ph/0103065] [INSPIRE].

[15] S. Lavignac, I. Masina and C.A. Savoy, $\tau \rightarrow \mu \gamma$ and $\mu \rightarrow e \gamma$ as probes of neutrino mass models, Phys. Lett. B 520 (2001) 269 [hep-ph/0106245] [INSPIRE].

[16] X.-J. Bi and Y.-B. Dai, Lepton flavor violation in the supersymmetric grand unified models with a lopsided mass matrix, Phys. Rev. D 66 (2002) 076006 [hep-ph/0112077] [INSPIRE].

[17] J.R. Ellis, J. Hisano, M. Raidal and Y. Shimizu, A new parametrization of the seesaw mechanism and applications in supersymmetric models, Phys. Rev. D 66 (2002) 115013 [hep-ph/0206110] [INSPIRE].

[18] F. Deppisch, H. Pas, A. Redelbach, R. Ruckl and Y. Shimizu, Probing the Majorana mass scale of right-handed neutrinos in mSUGRA, Eur. Phys. J. C 28 (2003) 365 [hep-ph/0206122] [INSPIRE].

[19] T. Fukuyama, T. Kikuchi and N. Okada, Lepton flavor violating processes and muon g-2 in minimal supersymmetric SO(10) model, Phys. Rev. D 68 (2003) 033012 [hep-ph/0304190] [INSPIRE].

[20] A. Brignole and A. Rossi, Anatomy and phenomenology of $\mu-\tau$ lepton flavor violation in the MSSM, Nucl. Phys. B 701 (2004) 3 [hep-ph/0404211] [INSPIRE].

[21] A. Masiero, S.K. Vempati and O. Vives, Massive neutrinos and flavor violation, New J. Phys. 6 (2004) 202 [hep-ph/0407325] [INSPIRE].

[22] T. Fukuyama, A. Ilakovac and T. Kikuchi, Lepton flavor violating leptonic/semileptonic decays of charged leptons in the minimal supersymmetric Standard Model,

Eur. Phys. J. C 56 (2008) 125 [hep-ph/0506295] [INSPIRE]. 
[23] S. Petcov, W. Rodejohann, T. Shindou and Y. Takanishi, The see-saw mechanism, neutrino Yukawa couplings, LFV decays $\ell_{i} \rightarrow \ell_{j}+\gamma$ and leptogenesis, Nucl. Phys. B 739 (2006) 208 [hep-ph/0510404] [INSPIRE].

[24] E. Arganda and M.J. Herrero, Testing supersymmetry with lepton flavor violating $\tau$ and $\mu$ decays, Phys. Rev. D 73 (2006) 055003 [hep-ph/0510405] [INSPIRE].

[25] F. Deppisch, H. Pas, A. Redelbach and R. Ruckl, Constraints on SUSY seesaw parameters from leptogenesis and lepton flavor violation, Phys. Rev. D 73 (2006) 033004 [hep-ph/0511062] [INSPIRE].

[26] C.E. Yaguna, Constraining mSUGRA parameters with $\mu \rightarrow e \gamma$ and $\mu$-e conversion in nuclei, Int. J. Mod. Phys. A 21 (2006) 1283 [hep-ph/0502014] [INSPIRE].

[27] L. Calibbi, A. Faccia, A. Masiero and S. Vempati, Lepton flavour violation from SUSY-GUTs: where do we stand for MEG, PRISM/PRIME and a super flavour factory, Phys. Rev. D 74 (2006) 116002 [hep-ph/0605139] [INSPIRE].

[28] S. Antusch, E. Arganda, M. Herrero and A. Teixeira, Impact of $\theta_{13}$ on lepton flavour violating processes within SUSY seesaw, JHEP 11 (2006) 090 [hep-ph/0607263] [INSPIRE].

[29] E. Arganda, M. Herrero and A. Teixeira, $\mu$-e conversion in nuclei within the CMSSM seesaw: universality versus non-universality, JHEP 10 (2007) 104 [arXiv:0707.2955] [INSPIRE].

[30] E. Arganda, M. Herrero and J. Portoles, Lepton flavour violating semileptonic $\tau$ decays in constrained MSSM-seesaw scenarios, JHEP 06 (2008) 079 [arXiv: 0803.2039] [INSPIRE].

[31] N. Arkani-Hamed, H.-C. Cheng, J.L. Feng and L.J. Hall, Probing lepton flavor violation at future colliders, Phys. Rev. Lett. 77 (1996) 1937 [hep-ph/9603431] [INSPIRE].

[32] I. Hinchliffe and F. Paige, Lepton flavor violation at the CERN LHC, Phys. Rev. D 63 (2001) 115006 [hep-ph/0010086] [InSPIRE].

[33] D. Carvalho, J.R. Ellis, M. Gomez, S. Lola and J. Romao, $\tau$ flavor violation in sparticle decays at the LHC, Phys. Lett. B 618 (2005) 162 [hep-ph/0206148] [INSPIRE].

[34] M.R. Buckley and H. Murayama, How can we test seesaw experimentally?, Phys. Rev. Lett. 97 (2006) 231801 [hep-ph/0606088] [INSPIRE].

[35] M. Hirsch, J. Valle, W. Porod, J. Romao and A. Villanova del Moral, Probing minimal supergravity in type-I seesaw with lepton flavour violation at the $\mathrm{LHC}$, Phys. Rev. D 78 (2008) 013006 [arXiv:0804.4072] [INSPIRE].

[36] E. Carquin, J. Ellis, M. Gomez, S. Lola and J. Rodriguez-Quintero, Search for $\tau$ flavour violation at the LHC, JHEP 05 (2009) 026 [arXiv:0812.4243] [INSPIRE].

[37] J. Esteves et al., Flavour violation at the LHC: type-I versus type-II seesaw in minimal supergravity, JHEP 05 (2009) 003 [arXiv:0903.1408] [INSPIRE].

[38] A.J. Buras, L. Calibbi and P. Paradisi, Slepton mass-splittings as a signal of LFV at the LHC, JHEP 06 (2010) 042 [arXiv:0912.1309] [INSPIRE].

[39] A. Abada, A. Figueiredo, J. Romao and A. Teixeira, Interplay of LFV and slepton mass splittings at the LHC as a probe of the SUSY seesaw, JHEP 10 (2010) 104 [arXiv: 1007.4833] [INSPIRE].

[40] A. Abada, A. Figueiredo, J. Romao and A. Teixeira, Probing the supersymmetric type-III seesaw: LFV at low-energies and at the LHC, JHEP 08 (2011) 099 [arXiv:1104.3962] [INSPIRE]. 
[41] L. Calibbi, R. Hodgkinson, J. Jones Perez, A. Masiero and O. Vives, Flavour and collider interplay for SUSY at LHC7, Eur. Phys. J. C 72 (2012) 1863 [arXiv:1111.0176] [INSPIRE].

[42] I. Galon and Y. Shadmi, Kinematic edges with flavor splitting and mixing, Phys. Rev. D 85 (2012) 015010 [arXiv: 1108.2220] [InSPIRE].

[43] C. Arbelaez, M. Hirsch and L. Reichert, Supersymmetric mass spectra and the seesaw type-I scale, JHEP 02 (2012) 112 [arXiv:1112.4771] [INSPIRE].

[44] A. Abada, A. Figueiredo, J. Romao and A. Teixeira, Lepton flavour violation: physics potential of a linear collider, JHEP 08 (2012) 138 [arXiv:1206.2306] [INSPIRE].

[45] M. Cannoni, J. Ellis, M.E. Gomez and S. Lola, Neutrino textures and charged lepton flavour violation in light of $\theta_{13}, M E G$ and LHC data, Phys. Rev. D 88 (2013) 075005 [arXiv: 1301.6002] [INSPIRE].

[46] T2K collaboration, K. Abe et al., Indication of electron neutrino appearance from an accelerator-produced off-axis muon neutrino beam, Phys. Rev. Lett. 107 (2011) 041801 [arXiv: 1106.2822] [INSPIRE].

[47] Double-CHOOZ collaboration, Y. Abe et al., Indication for the disappearance of reactor electron antineutrinos in the Double CHOOZ experiment, Phys. Rev. Lett. 108 (2012) 131801 [arXiv:1112.6353] [INSPIRE].

[48] RENO collaboration, J. Ahn et al., Observation of reactor electron antineutrino disappearance in the RENO experiment, Phys. Rev. Lett. 108 (2012) 191802 [arXiv: 1204.0626] [INSPIRE].

[49] Daya Bay collaboration, F. An et al., Improved measurement of electron antineutrino disappearance at Daya Bay, Chin. Phys. C 37 (2013) 011001 [arXiv:1210.6327] [InSPIRE].

[50] ATLAS collaboration, Search for squarks and gluinos with the ATLAS detector in final states with jets and missing transverse momentum and $20.3 \mathrm{fb}^{-1}$ of $\sqrt{s}=8 \mathrm{TeV}$ proton-proton collision data, ATLAS-CONF-2013-047, CERN, Geneva Switzerland (2013).

[51] CMS collaboration, Search for top-squark pair production in the single lepton final state in pp collisions at $8 \mathrm{TeV}$, CMS-PAS-SUS-13-011, CERN, Geneva Switzerland (2013).

[52] ATLAS collaboration, Search for direct production of the top squark in the all-hadronic $t \bar{t}+E_{\mathrm{T}}^{\mathrm{miss}}$ final state in $21 \mathrm{fb}^{-1}$ of pp collisions at $\sqrt{s}=8 \mathrm{TeV}$ with the ATLAS detector, ATLAS-CONF-2013-024, CERN, Geneva Switzerland (2013).

[53] ATLAS collaboration, Search for direct third generation squark pair production in final states with missing transverse momentum and two b-jets in $\sqrt{s}=8 \mathrm{TeV}$ pp collisions with the ATLAS detector, ATLAS-CONF-2013-053, CERN, Geneva Switzerland (2013).

[54] ATLAS collaboration, Search for strongly produced superpartners in final states with two same sign leptons with the ATLAS detector using $21 \mathrm{fb}^{-1}$ of proton-proton collisions at $\sqrt{s}=8 \mathrm{TeV}$., ATLAS-CONF-2013-007, CERN, Geneva Switzerland (2013).

[55] CMS collaboration, Search for direct EWK production of SUSY particles in multilepton modes with $8 \mathrm{TeV}$ data, CMS-PAS-SUS-12-022, CERN, Geneva Switzerland (2012).

[56] ATLAS collaboration, Search for electroweak production of supersymmetric particles in final states with at least two hadronically decaying taus and missing transverse momentum with the ATLAS detector in proton-proton collisions at $\sqrt{s}=8 \mathrm{TeV}$, ATLAS-CONF-2013-028, CERN, Geneva Switzerland (2013). 
[57] ATLAS collaboration, Search for direct-slepton and direct-chargino production in final states with two opposite-sign leptons, missing transverse momentum and no jets in $20 \mathrm{fb}^{-1}$ of pp collisions at $\sqrt{s}=8 \mathrm{TeV}$ with the ATLAS detector, ATLAS-CONF-2013-049, CERN, Geneva Switzerland (2013).

[58] CMS collaboration, Searches for long-lived charged particles in pp collisions at $\sqrt{s}=7$ and $8 \mathrm{TeV}$, arXiv:1305.0491 [INSPIRE].

[59] ATLAS collaboration, Observation of a new particle in the search for the Standard Model Higgs boson with the ATLAS detector at the LHC, Phys. Lett. B 716 (2012) 1 [arXiv: 1207.7214] [INSPIRE].

[60] ATLAS collaboration, Combined measurements of the mass and signal strength of the Higgs-like boson with the ATLAS detector using up to $25 \mathrm{fb}^{-1}$ of proton-proton collision data, ATLAS-CONF-2013-014, CERN, Geneva Switzerland (2013).

[61] ATLAS collaboration, Combined coupling measurements of the Higgs-like boson with the ATLAS detector using up to $25 \mathrm{fb}^{-1}$ of proton-proton collision data, ATLAS-CONF-2013-034, CERN, Geneva Switzerland (2013).

[62] CMS collaboration, Observation of a new boson with mass near $125 \mathrm{GeV}$ in pp collisions at $\sqrt{s}=7$ and $8 \mathrm{TeV}$, JHEP 06 (2013) 081 [arXiv: 1303.4571] [INSPIRE].

[63] MEG collaboration, J. Adam et al., New constraint on the existence of the $\mu^{+} \rightarrow e^{+} \gamma$ decay, Phys. Rev. Lett. 110 (2013) 201801 [arXiv:1303.0754] [inSPIRE].

[64] R.-D. Heuer, News from CERN, LHC status and strategy for linear colliders, arXiv: 1202.5860 [INSPIRE].

[65] ATLAS collaboration, Searches for supersymmetry at the high luminosity LHC with the ATLAS detector, ATL-PHYS-PUB-2013-002, CERN, Geneva Switzerland (2013).

[66] L. Calibbi, D. Chowdhury, A. Masiero, K. Patel and S. Vempati, Status of supersymmetric type-I seesaw in $\mathrm{SO}(10)$ inspired models, JHEP 11 (2012) 040 [arXiv:1207.7227] [INSPIRE].

[67] Y. Grossman and H.E. Haber, Sneutrino mixing phenomena, Phys. Rev. Lett. 78 (1997) 3438 [hep-ph/9702421] [INSPIRE].

[68] M. Raidal et al., Flavour physics of leptons and dipole moments, Eur. Phys. J. C 57 (2008) 13 [arXiv:0801.1826] [INSPIRE].

[69] M. Arana-Catania, S. Heinemeyer and M. Herrero, New constraints on general slepton flavor mixing, Phys. Rev. D 88 (2013) 015026 [arXiv:1304.2783] [INSPIRE].

[70] BABAR collaboration, B. Aubert et al., Searches for lepton flavor violation in the decays $\tau^{ \pm} \rightarrow e^{ \pm} \gamma$ and $\tau^{ \pm} \rightarrow \mu^{ \pm} \gamma$, Phys. Rev. Lett. 104 (2010) 021802 [arXiv:0908. 2381] [INSPIRE].

[71] I. Hinchliffe, F. Paige, M. Shapiro, J. Soderqvist and W. Yao, Precision SUSY measurements at CERN LHC, Phys. Rev. D 55 (1997) 5520 [hep-ph/9610544] [INSPIRE].

[72] B. Allanach, C. Lester, M.A. Parker and B. Webber, Measuring sparticle masses in nonuniversal string inspired models at the LHC, JHEP 09 (2000) 004 [hep-ph/0007009] [INSPIRE].

[73] H. Bachacou, I. Hinchliffe and F.E. Paige, Measurements of masses in SUGRA models at CERN LHC, Phys. Rev. D 62 (2000) 015009 [hep-ph/9907518] [INSPIRE]. 
[74] M. Gonzalez-Garcia, M. Maltoni, J. Salvado and T. Schwetz, Global fit to three neutrino mixing: critical look at present precision, JHEP 12 (2012) 123 [arXiv:1209.3023] [INSPIRE].

[75] W. Porod, SPheno, a program for calculating supersymmetric spectra, SUSY particle decays and SUSY particle production at $e^{+} e^{-}$colliders, Comput. Phys. Commun. 153 (2003) 275 [hep-ph/0301101] [INSPIRE].

[76] W. Porod and F. Staub, SPheno 3.1: extensions including flavour, CP-phases and models beyond the MSSM, Comput. Phys. Commun. 183 (2012) 2458 [arXiv:1104.1573] [inSPIRE].

[77] P. Bechtle et al., Recent developments in HiggsBounds and a preview of HiggsSignals, PoS (CHARGED 2012) 024 [arXiv: 1301.2345] [INSPIRE].

[78] Particle Data Group collaboration, J. Beringer et al., Review of particle physics (RPP), Phys. Rev. D 86 (2012) 010001 [InSPIRE].

[79] G. Bélanger, F. Boudjema, A. Pukhov and A. Semenov, MicrOMEGAs3.1: a program for calculating dark matter observables, arXiv:1305.0237 [INSPIRE].

[80] J. Camargo-Molina, B. O'Leary, W. Porod and F. Staub, Stability of the CMSSM against sfermion VEVs, arXiv:1309.7212 [INSPIRE].

[81] MEGA collaboration, M. Brooks et al., New limit for the family number nonconserving decay $\mu^{+} \rightarrow e^{+} \gamma$, Phys. Rev. Lett. 83 (1999) 1521 [hep-ex/9905013] [InSPIRE].

[82] A. Baldini et al., MEG upgrade proposal, arXiv:1301.7225 [INSPIRE].

[83] Planck collaboration, P. Ade et al., Planck 2013 results. XV. CMB power spectra and likelihood, arXiv:1303.5075 [INSPIRE].

[84] WMAP collaboration, G. Hinshaw et al., Nine-year Wilkinson Microwave Anisotropy Probe (WMAP) observations: cosmological parameter results, Astrophys. J. Suppl. 208 (2013) 19 [arXiv: 1212.5226] [INSPIRE].

[85] G. Gelmini, P. Gondolo, A. Soldatenko and C.E. Yaguna, The effect of a late decaying scalar on the neutralino relic density, Phys. Rev. D 74 (2006) 083514 [hep-ph/0605016] [InSPIRE].

[86] G.B. Gelmini and P. Gondolo, Neutralino with the right cold dark matter abundance in (almost) any supersymmetric model, Phys. Rev. D 74 (2006) 023510 [hep-ph/0602230] [INSPIRE].

[87] W. Beenakker, R. Hopker, M. Spira and P. Zerwas, Squark and gluino production at hadron colliders, Nucl. Phys. B 492 (1997) 51 [hep-ph/9610490] [INSPIRE].

[88] W. Beenakker et al., The production of charginos/neutralinos and sleptons at hadron colliders, Phys. Rev. Lett. 83 (1999) 3780 [Erratum ibid. 100 (2008) 029901] [hep-ph/9906298] [INSPIRE].

[89] $\mu 2 e$ collaboration, A. Gaponenko, The $\mu 2 e$ experiment: a new high-sensitivity muon to electron conversion search at Fermilab, FERMILAB-CONF-12-490-PPD, Fermilab, Batavia U.S.A. (2012) [INSPIRE].

[90] COMET collaboration, Y. Kuno, A search for muon-to-electron conversion at J-PARC: the COMET experiment, Prog. Theor. Exp. Phys. 2013 (2013) 022C01 [inSPIRE].

[91] R. Barlow, The PRISM/PRIME project, Nucl. Phys. Proc. Suppl. 218 (2011) 44 [inSPIRE]. 